Article

\title{
Design of Multilayer Ring Emitter Based on Metamaterial for Thermophotovoltaic Applications
}

\author{
Fekadu Tolessa Maremi ${ }^{1}{ }^{\circledR}$, Namkyu Lee ${ }^{1}$, Geehong Choi ${ }^{1}$, Taehwan Kim ${ }^{2}$ \\ and Hyung Hee Cho ${ }^{1, *}$ \\ 1 Department of Mechanical Engineering, Yonsei University, Seoul 03722, Korea; \\ 21_mylove@yonsei.ac.kr (F.T.M); motivebuzz@yonsei.ac.kr (N.L); or50341@yonsei.ac.kr (G.C) \\ 2 Semiconductor R\&D Center, Samsung Electronics Inc., Suwon 18448, Korea; kimth0610@yonsei.ac.kr \\ * Correspondence: hhcho@yonsei.ac.kr; Tel.: +82-2-2123-2828
}

Received: 8 August 2018; Accepted: 29 August 2018; Published: 31 August 2018

check for updates

\begin{abstract}
The objective of this study is to design a broadband and wide-angle emitter based on metamaterials with a cut-off wavelength of $2.1 \mu \mathrm{m}$ to improve the spectral efficiency of thermophotovoltaic emitters. To obtain broadband emission, we conducted the geometric parameter optimization of the number of stacked layers, the inner and outer radii of the nano-rings, and the thickness of the nano-rings. The numerical simulation results showed that the proposed emitter had an average emissivity of 0.97 within the targeted wavelength, which ranged from $0.2 \mu \mathrm{m}$ to $2.1 \mu \mathrm{m}$. In addition, the presented multilayer nano-ring emitter obtained $79.6 \%$ spectral efficiency with an InGaAs band gap of $0.6 \mathrm{eV}$ at $1400 \mathrm{~K}$.
\end{abstract}

Keywords: thermophotovoltaic; selective emitter; broadband absorber; metamaterial

\section{Introduction}

The conversion of solar and waste heat energies into electricity is a promising energy-harvesting technology due to the abundance, omnipresence, and bio-friendly properties of these energy sources. Thermophotovoltaic (TPV) devices harvest electromagnetic energy and convert it to electrical energy [1]. A heat source, a thermal radiator, and photovoltaic (PV) cells are the fundamental parts of a TPV system. The role of the thermal radiator is to convert solar or waste heat energies into thermal radiation, from which a PV cell generates electricity using the photovoltaic effect [2]. However, the conversion of electromagnetic energy to electrical power happens only when the photon energy is higher than the energy band gap of the PV cell. To maximize the power of electricity generation and increase the efficiency of TPV systems, the energy of photons should be higher than the band gap of the PV cell. For this reason, the selective emitter should be a perfect broadband and wide-angle emissivity above the band gap energy of the PV cell. If the TPV emitter has high emissivity within the targeted wavelength region and low emissivity above cut-off wavelength, the PV cell can effectively operate to generate electrical power with relatively low thermal loss [3-5].

TPV emitters can be designed in 1D [1,6,7], 2D [8], as grating and 3D photonic crystals [9-11], or from artificial optical materials known as metamaterials. Metamaterials contain repeating patterns at small scales and have properties that have not yet been found in nature [12,13]. Metamaterial emitters are typically designed with different symmetry structures such as disks [14,15], squares [16-18], crosses [19-21], pyramids [22-25], nanowires [26,27], cones [28], and nanoparticles [3] to increase TPV performance achieved by perfect emissivity above the energy band gap of PV cell. Similarly, the nano-sized ring has been studied for various applications because of high sensitivity and multiplexing capability [29]. However, there has been less consideration of a nano-sized ring for TPV emitters. For this reason, it is important to design the nano-sized ring emitter with consideration to cut-off wavelength to maximize energy conversion efficiency. 
In this study, we designed an emitter based on metamaterials to enhance a perfect broadband and wide-angle absorber within the energy band gap of InGaAs (which had cut-off wavelength of $2.1 \mu \mathrm{m}$ to increase the efficiency of the TPV system). Based on numerical simulation, we optimized a multilayered nano-ring structure on the basis of proposed metamaterials such as number of layers, radius and thickness of the ring. Near perfect and wide-angle emissivity were obtained at the target wavelength (visible and near-IR), which matched with the InGaAs cut-off wavelength at $2.1 \mu \mathrm{m}$. In addition, we obtained $79.6 \%$ spectral efficiency of the emitter at $1400 \mathrm{~K}$ and enhanced efficiency of the TPV system.

\section{Research Methods}

Figure 1 shows the proposed metamaterial structure comprising tungsten $(\mathrm{W})$ and aluminum nitride (AlN) multilayer rings (n-layers), with a top layer of $W$, where $n$ is the number of $W$ and AlN layers due to high operation temperature $(<2000 \mathrm{~K})$. W has been used for high temperature applications due to a high melting temperature $(3722 \mathrm{~K})$. AlN, too, has sufficient melting temperature $(2500 \mathrm{~K})$ and, in particular, good oxidation resistance [30,31]. Thus, $\mathrm{W}$ and AlN are suitable for fabrication. A unit cell has a stacked layer, designed with strips of $W$ and AlN with height $h_{1}$ from nano-rings with a periodic distance $\Lambda$, an inner radius $r_{2}$ and an outer radius $r_{1}$. A thin film of AlN, thickness $h_{2}$, is designed on top of a W film of thickness $h_{3}$, which acts as a perfect reflector. The width of the unit cell was $\Lambda=400 \mathrm{~nm}$, the thickness of the AlN and W film were $h_{2}=40 \mathrm{~nm}$ and $h_{3}=120 \mathrm{~nm}$, respectively, and these parameters were fixed in this study. The variables were the number of staked layers $(\mathrm{n})$, inner $\left(\mathrm{r}_{1}\right)$ and outer radius $\left(\mathrm{r}_{2}\right)$, and thickness of the nano-ring layer $\left(\mathrm{h}_{1}\right)$.

Numerical simulation was conducted on the unit cell with $\Lambda=400 \mathrm{~nm}$ using a three-dimensional electro-magnetic wave and the wavelength domain study was performed using COMSOL Multiphysics software. During the numerical analysis, we assigned the perfectly matched layer on the top and bottom of the computational domains to minimize reflection. The periodic boundary condition was also set at both the right and left boundaries to account for an infinite flat surface on the emitter. The electromagnetic waves on the emitter were considered to be incident plane waves. The refractive index (optical properties) of the metallic film (W) and the dielectric spacer (AlN) was taken from [32,33], respectively.

During the numerical analysis, we considered two ports. The first port was known as the in-port, which produced electromagnetic waves, and the second port was called the out-port, which transmitted the incident wave. From the simulation results, we gathered the Scattering parameters (S-parameters). We assume that no transmittance occurred, as the back of the reflector was made by W. Thus, we can calculate the reflection coefficient based on the S-parameter of $S_{11} ; S_{11}$ meant the signal ratio between the incident wave and the reflected wave. Consequently, we can calculate reflectivity as:

$$
\rho=1-|\Gamma|^{2}=1-\left|S_{11}\right|^{2}
$$

where $\rho$ and $\Gamma$ are reflectivity and reflection coefficient, respectively. In addition, we also derive the absorptivity and emissivity based on Kirchhoff's law with the assumption of no transmittance as follows:

$$
\varepsilon(\lambda)=\alpha(\lambda)=1-\rho(\lambda)
$$

Finally, we evaluate the emissivity based on absorptivity from the simulation results.

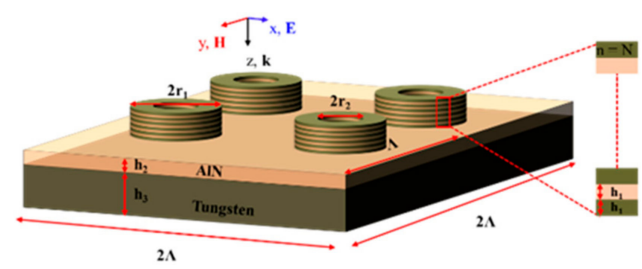

Figure 1. Schematic representation of the designed metamaterial structure. 


\section{Result and Discussion}

\subsection{Effect of Number of Layers ( $n)$ and Outer Radius $\left(r_{1}\right)$}

Figure 2 showed the effect of number of stack layers $(n)$ and the outer radius of the strips $\left(\mathrm{r}_{1}\right)$ on the spectral emissivity of a selective emitter. As shown in Figure 2, two peaks occurred in the case of a single ring layer: the first peak was found at a wavelength of $0.5 \mu \mathrm{m}$ and the second peak at a wavelength of $2.8 \mu \mathrm{m}$. In Figure 2a, as $n$ increased, the first peak was slightly changed and the second peak shifted to lower wavelengths. This result suggested that the stack layer number influences and maximizes the emissivity for the second peak. The widest and most near perfect broadband emissivity occurs when $n=11$, (red line) at $r_{1}=120$ and $h_{1}=15 \mathrm{~nm}$ for each layer of nano-ring. Figure $2 b$ showed the dependence of the outer radius $r_{1}$ on the emissivity. As can be seen, when radius $r_{1}$ was increased from $90 \mathrm{~nm}$ to $130 \mathrm{~nm}$, the emissivity spectra of the second peak was maximized and was a near perfect broadband. Our results revealed that when the number of layers was 11 and the outer radius was $120 \mathrm{~nm}$, perfect broadband and emission with consideration of cut-off wavelength was achieved.

To explain the perfect broadband absorption, we analyzed the electromagnetic field around the multilayer ring emitter. Several excitation mechanisms occurred with resonance frequencies; here, we considered magnetic polariton. This excitation method can powerfully localize the electromagnetic energy in the dielectric (AlN) layer, which was inserted between the first $\mathrm{W}$ ring layer and the ground W film layer, as well as between two consecutive metal (W) layers in a ring (metal-dielectric-metal or $\mathrm{W}-\mathrm{AlN}-\mathrm{W}$ ). Due to the existence of free charge on the thin film $\mathrm{W}$ surfaces of the ground layer, the first $\mathrm{W}$ ring layer, or between two sub-layers in the nano-rings, there was a net flow of oscillating electric currents, which resulted in induced magnetic fields. This mechanism was known as the excitation of magnetic polaritons (MPs) [34]. The simultaneous collaboration of magnetic polariton (MP) resonances at a targeted wavelength created a near perfect broadband emissivity.

The origin of the electromagnetic property of a metamaterial came from the arrangement of the periodic unit cell and its geometry. The electromagnetic properties can be controlled by changing the parameters of the $\mathrm{W}$ and AlN strips, their pitch, and the number of layers. Conducting particles with a resonance size of the order $\lambda / 10$ were well known, where $\lambda$ was the wavelength of the electromagnetic wave [35]. Our design contained a multilayer structure, through which we can achieve resonances at different wavelengths due to the MPs. The combination of these resonances enhanced broadband emissivity. A nano-ring and multilayer selective metamaterial emitter design were chosen for polarization-independent broadband emissivity.

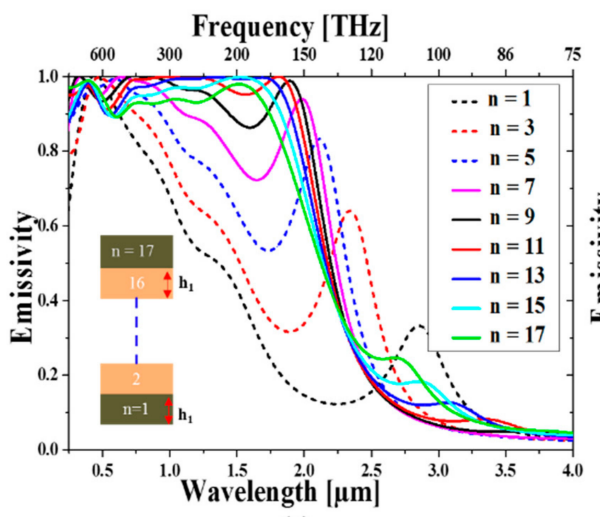

(a)

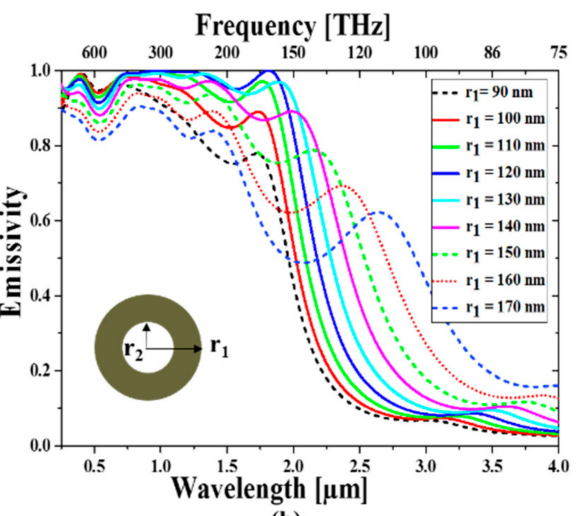

(b)

Figure 2. The emissivity spectra of the proposed metamaterial at (a) the variation of number of ring layers $n$ at $r_{1}=120 \mathrm{~nm}$ and (b) the change of the outer radius $r_{1}$ at $n=11$. Inset in (a) is a side view of the number of layers and in (b) it is the top view. 
Figure 3a-c illustrated the distribution of the normalized energy flow at wavelengths of 1.9, 2.1, and $5 \mu \mathrm{m}$, respectively. A traveling electromagnetic wave in space carries energy and its energy density is related to electric and magnetic fields. The amount of energy flowing through a given unit area in the perpendicular direction to the incident energy per unit of time is called the Poynting vector $\left(S=\operatorname{Re}\left(E \times \mathbf{H}^{*}\right) / 2\right)$ [22], where $S$ is poynting vector, $\operatorname{Re}$ is real, $\mathbf{E}$ is electric field and $\mathbf{H}^{*}$ is complex conjugate of magnetic field. As shown in Figure 3, we recognized that the pointing vector field was matched with the magnitude of emissivity. The results showed high-energy density distribution at high emission and weak-energy density distribution at low emission.

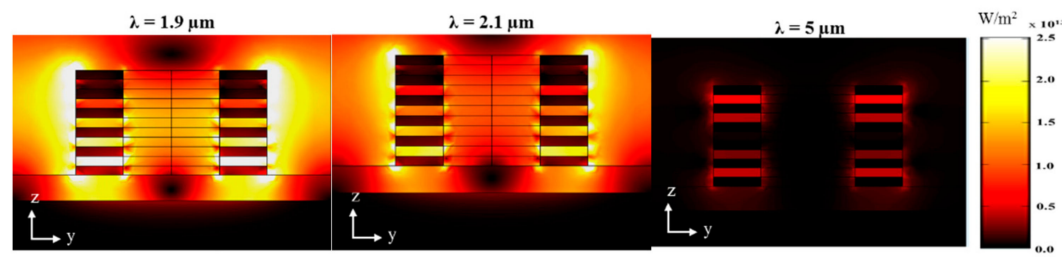

(a)

(b)

(c)

Figure 3. The distribution of energy flow (Poynting vector) at wavelengths of $1.9 \mu \mathrm{m}, 2.1 \mu \mathrm{m}$ and $5 \mu \mathrm{m}$ within a multilayer nano-ring: The results show that high spectral emissivity is close relation with high energy density distribution: (a) $\varepsilon(\lambda)=0.96$, (b) $\varepsilon(\lambda)=0.63$ and (c) $\varepsilon(\lambda)=0$.

\subsection{Effect of Inner Radius $\left(r_{2}\right)$ and Thickness of Nano-Ring $\left(h_{1}\right)$}

As explained in Section 3.1, metamaterial properties are effectively related to geometry, such as thickness of the dielectric and inner radius [28]. Figure 4 illustrates the effect of the inner radius $r_{2}$ and the thickness of the nano-ring $\mathrm{h}_{1}$, on the spectral emissivity of the designed selective emitter. The results showed that the increase of $r_{2}$ changed the peak from $1.7 \mu \mathrm{m}$ to $2.3 \mu \mathrm{m}$; the changed peak caused a weaker interaction between peaks and lower emissivity around $1.7 \mu \mathrm{m}$. It meant that $\mathrm{r}_{2}$ had a close relation with the peak around $1.7 \mu \mathrm{m}$ and $2.3 \mu \mathrm{m}$ and maintained broadband emissivity below $2.1 \mu \mathrm{m}$. The dependence of the emissivity spectra on $h_{1}$ is illustrated in Figure $4 b$. When $h_{1}$ increased from $10 \mathrm{~nm}$ to $30 \mathrm{~nm}$, the broadband emissivity increased as value of $h_{1}$ increased from $10 \mathrm{~nm}$ to $15 \mathrm{~nm}$ and at that point decreased as $h_{1}$ increased from $20 \mathrm{~nm}$ to $30 \mathrm{~nm}$. This result showed that a near perfect broadband emitter can be obtained when $\mathrm{h}_{1}$ $=15 \mathrm{~nm}$ (red line) and $\mathrm{r}_{2}=60 \mathrm{~nm}$ to yield broadband emissivity with an average of about $96.6 \%$ in the wavelength region of $0.2 \mu \mathrm{m}$ to $2.1 \mu \mathrm{m}$. The excitation of MP depends on the radius and thickness of the dielectric layer [36]. The possible reason for enhanced broadband emissivity in the targeted wavelength is the combination of excitation MP of multiple resonances. Finally, near perfect broadband emission was obtained at target wavelengths from an optimized geometry of $n=11, r_{1}=120 \mathrm{~nm}, r_{2}=60 \mathrm{~nm}$, and $h_{1}=15 \mathrm{~nm}$.

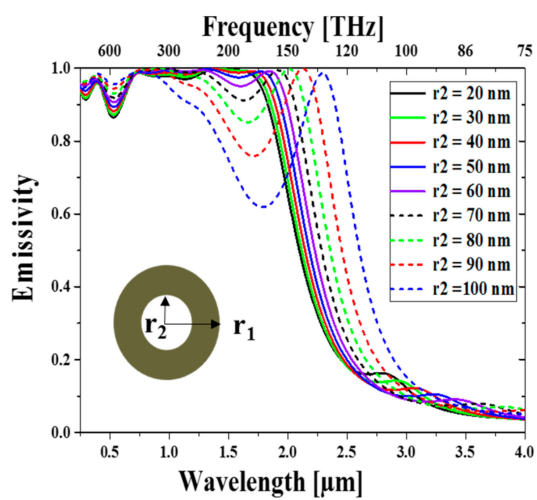

(a)

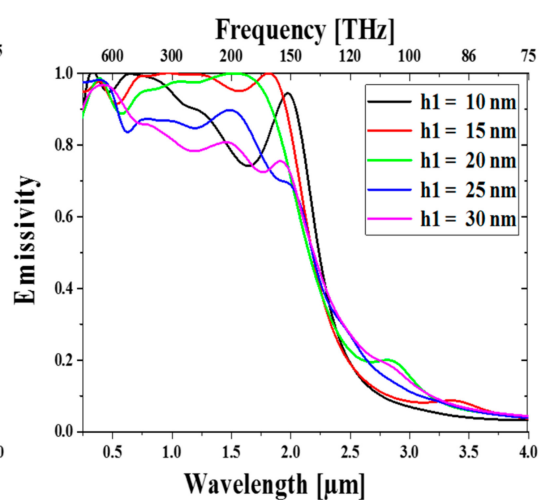

(b)

Figure 4. The influence of geometry on the emissivity at (a) various inner radius $r_{2}$ at $h_{1}=15 \mathrm{~nm}$, $\mathrm{h}_{2}=40 \mathrm{~nm}, \mathrm{~h}_{3}=120 \mathrm{~nm}, \mathrm{r}_{1}=120 \mathrm{~nm}, \mathrm{n}=11$ and $\Lambda=400 \mathrm{~nm}$. Inset in (a) shows the top view of nano-ring, (b) various thickness of nano-ring $\mathrm{h}_{1}$ at $\mathrm{h}_{2}=40 \mathrm{~nm}$. 


\subsection{Angular Emissivity}

The behavior of angular emissivity of TPV emitter is vital due to $x-y$ polarizations angles of electromagnetic waves [36]. We studied the effect of $x-y$ polarizations and azimuthal angles on spectral emissivity of the proposed selective emitter at a normal polar angle $(\theta=0)$. However, the results show that the designed metamaterial is independent $x-y$ polarizations and azimuthal angles. This shows that the proposed emitter is symmetric (nano-ring), which means that the polarization is independent. Figure 5 showed optimized emissivity spectra, as the angular emissivity varied from $0^{\circ}$ to $60^{\circ}$ in $15^{\circ}$ steps. The emissivity spectra at all angles were similar, but at $\theta=60^{\circ}$, some differences were noted. The possible reason of obtained wide-angle spectral emissivity was the excitation of MPs. The average emissivity at polar angle $\theta=60^{\circ}$ was above $89.5 \%$ in the wavelength range of $0.2 \mu \mathrm{m}$ to $2.1 \mu \mathrm{m}$, but decreased dramatically beyond the cut-off wavelength. These emission spectra were well matched with the external quantum efficiency (EQE) of InGaAs.

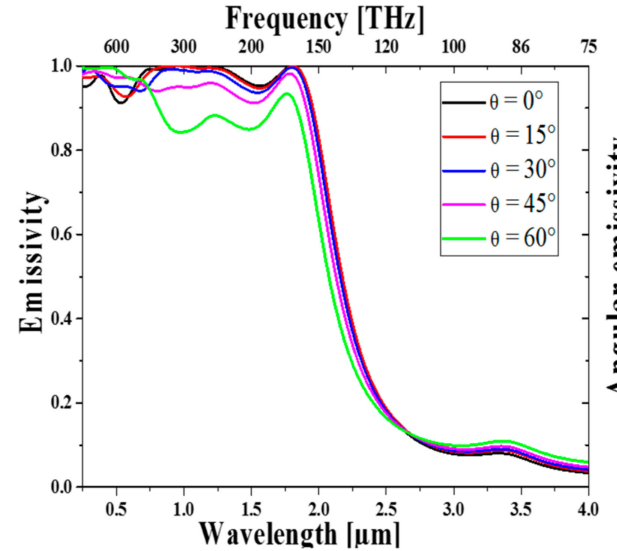

(a)

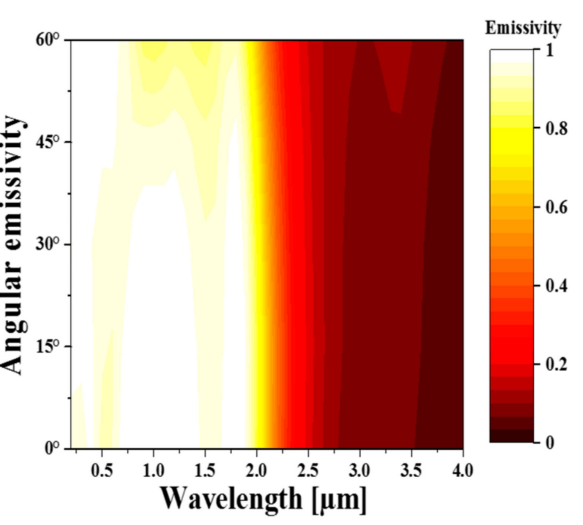

(b)

Figure 5. The emissivity of the proposed metamaterial at different angular emissivity, with dimensions of $\mathrm{h}_{1}=15 \mathrm{~nm}, \mathrm{~h}_{2}=40 \mathrm{~nm}, \mathrm{~h}_{3}=120 \mathrm{~nm}, \mathrm{r}_{1}=120 \mathrm{~nm}, \mathrm{r}_{2}=60 \mathrm{~nm}, \mathrm{n}=11$ and $\Lambda=400 \mathrm{~nm}$ : (a) as lines graph; (b) as 2-D color fill.

\subsection{Performance of TPV}

Figure 6 illustrates a comparison of the emissivity spectra of the proposed multilayer nano-ring metamaterial with other different thermal emitter metamaterials, wherein the shaded area indicates the EQE of InGaAs (0.6 eV) [37]. The blue line represents the spectrum emissivity of the proposed selective emitter, whereas other recent results are shown as a red dashed line, Woolf, et al. [20] and black dashed line, Zhao, et al. [16]. As can be clearly seen, we obtained a near perfect broadband emitter above the energy band gap of InGaAs, which dramatically decreased after the cut-off wavelength of $2.1 \mu \mathrm{m}$ (blue line). Due to the existence of a band gap in the PV cell, radiation energy below the band gap was considered as waste energy, while low radiation energy above bandgap was mandatory (convertible) energy.

The red and black dashed lines showing spectral emissivity in the figure were designed as an InGaAs PV cell with cut-off wavelength of $2.1 \mu \mathrm{m}$; the red dashed line indicates a nearly perfect broadband emissivity of wavelength less than $1.4 \mu \mathrm{m}$ and at that point it dramatically falls, whereas the black line shows broadband emissivity but not perfect near infrared wavelength. Nearly perfect broadband emissivity of $2.1 \mu \mathrm{m}$ wavelength enhanced the high spectral efficiency of the TPV emitter. The average spectral emissivity at a wavelength range of $0.2 \mu \mathrm{m}$ to $2.1 \mu \mathrm{m}$ band gap of InGaAs PV cell was about $96.6 \%, 79.7 \%$ and $81.7 \%$ for the blue, red dashed and black dashed lines, respectively, showing the performance characteristics of the designed nano-ring to be superior to the preceding work (red and black dashed line). Thus, our optimized emitter had a high performance. 


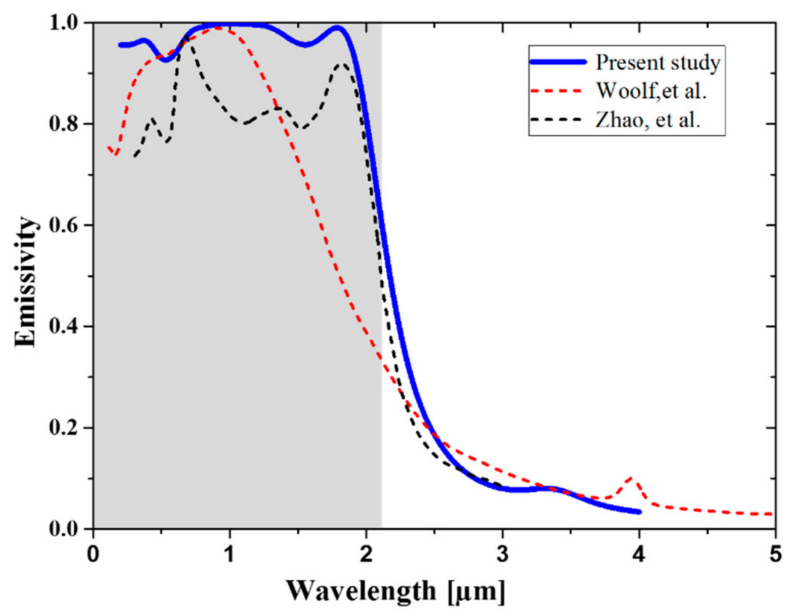

Figure 6. Comparison of the spectral emissivity of the proposed metamaterial (blue line), the external quantum efficiency (EQE) of InGaAs thermophotovoltaic (TPV) cells (shaded area), and results from some recent papers $([16,20])$ compared to other researches on emitters for In-GaAs photovoltaic (PV) cell.

To our knowledge, the cut-off wavelength, perfect emissivity below cut-off wavelength, and low emissivity above cut-off wavelength are the factors that require emphasis when designing an emitter, especially to increase the performance of a TPV system. In this study, we designed a multilayer ring metamaterial that offers better spectral efficiency performance in a TPV system.

To evaluate the efficiency of an optimized emitter for a TPV cell, we analyze the efficiency of the TPV system. The efficiency of a TPV system to convert radiated heat to electrical power is given by Equation (3) [3,22]:

$$
\eta_{T P V}=\eta_{S P} \eta_{P V}=\frac{P_{\text {out }}}{P_{\text {rad }}}
$$

where $\eta_{S P}$ is the spectra efficiency of the emitter, $\eta_{S P}$ is the efficiency of the photovoltaic cell, $P_{\text {out }}$ is the maximum output power, and $P_{\text {rad }}$ is the total radiant power from an emitter.

Spectral efficiency $\eta_{S P}$ is defined by Equation (4) [38]:

$$
\eta_{S P}=\frac{\int_{0}^{\lambda_{c}} E(\lambda) \varepsilon(\lambda) d \lambda}{\int_{0}^{\infty} E(\lambda) \varepsilon(\lambda) d \lambda}
$$

where $\lambda_{c}$ is the cut-off wavelength, $E(\lambda)$ is emission spectrum of a blackbody, and $\varepsilon(\lambda)$ is spectral emissivity of the proposed selective emitter metamaterial.

We extended our study of the optimized multilayer selective emitter with a black body at different temperatures to calculate the spectral efficiency of both the designed selective emitter and the black body. Spectral efficiency can be calculated using Equation (4). The best efficiency is achieved when the selective emitter has perfect emissivity above the band gap energy and low emissivity below the band gap energy. Figure 7a shows a comparison of the spectral irradiance of the black body with the selective emitter metamaterial at temperatures from $900 \mathrm{~K}$ to $1300 \mathrm{~K}$. Figure $7 \mathrm{~b}$ shows a histogram as the percentage of the spectral efficiency of the black body (black) and the selective emitter (blue) at different temperatures. As the temperature increased, the spectral efficiency also in-creased; this indicated that the emission peak shifted to a lower wavelength as the temperature rose, which showed that the emission spectrum peak was above the band gap region. The optimized design of the emitter had $\mathrm{h}_{1}=15 \mathrm{~nm}, \mathrm{~h}_{2}=40 \mathrm{~nm}, \mathrm{~h}_{3}=120 \mathrm{~nm}, \mathrm{r}_{1}=120 \mathrm{~nm}, \mathrm{r}_{2}=60 \mathrm{~nm}$ and 11 layers of nano-rings and achieved high efficiency of TPV. Table 1 shows the spectral efficiency of TPV cell InGaAs at different temperatures $(1000 \mathrm{~K}, 1200 \mathrm{~K}$, and $1400 \mathrm{~K})$ for single-layer and multilayer versions of the proposed selective emitter metamaterial. 


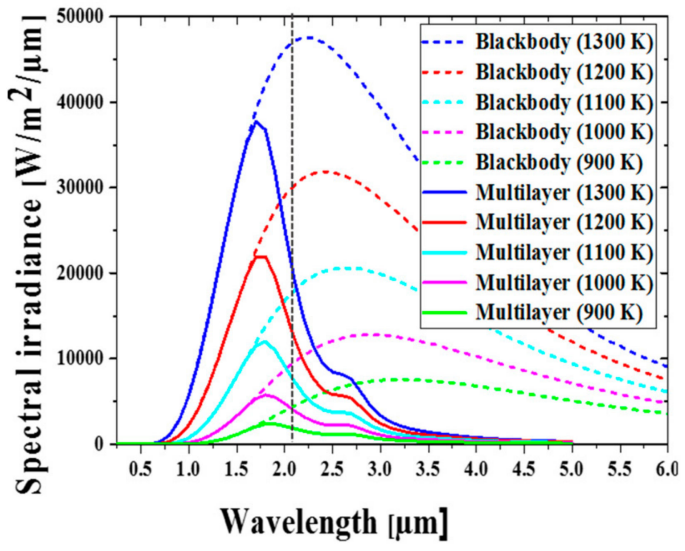

(a)

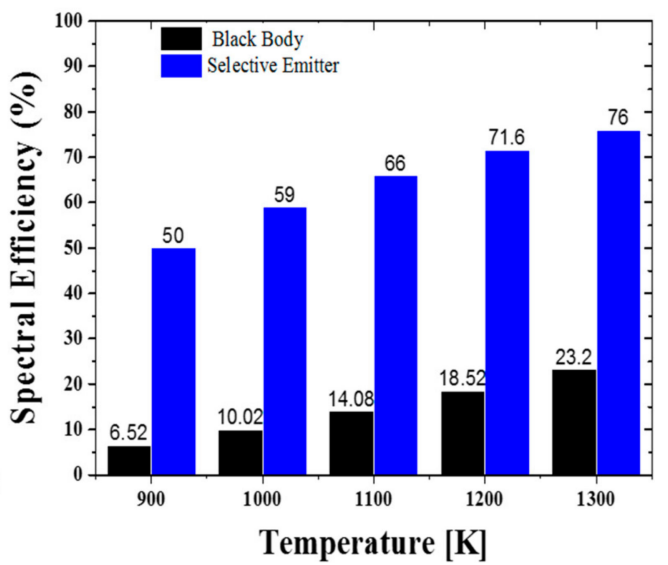

(b)

Figure 7. Comparison of the spectral irradiance and the spectral efficiency of a black body with the designed selective emitter at 900 K, 1000 K, 1100 K, 1200 K, and 1300 K. (a) Spectral irradiance of the proposed emitter was high radiation in the target wavelength and low radiation above the cut-off wavelength. (b) High spectral efficiency was obtained. For instance, $6.52 \%$ of radiated energies were convertible at $900 \mathrm{~K}$ by a black body; however, $50 \%$ of the radiated energies were convertible by designed selective emitter at $900 \mathrm{~K}$.

The results indicate a spectral efficiency $\left(\eta_{S P}\right) 76 \%$ and $82.4 \%$ for InGaAs with a $0.6 \mathrm{eV}$ band gap, comparing that Woolf et al. [20] achieved $43 \%$ and $52 \%$ spectral efficiency at $1300 \mathrm{~K}$ and $1500 \mathrm{~K}$, respectively. This demonstrates that excellent spectral efficiency is required to enhance efficiency of TPV systems. We also calculated the spectral efficiency of the proposed emitter from 0.2 to $15 \mu \mathrm{m}$ to checked error beyond $5 \mu \mathrm{m}$ wavelength. The difference was less than $1 \%$ of the spectral efficiency, and thus wavelength above $5 \mu \mathrm{m}$ was neglected; however, the spectral efficiency of the black body was higher than $1 \%$ beyond the wavelength.

Table 1. Spectral efficiency of single-layer and multilayer.

\begin{tabular}{ccc}
\hline \multirow{2}{*}{ Temp. $[\mathrm{K}]$} & \multicolumn{2}{c}{ InGaAs $\left(\lambda_{c}=\mathbf{2 . 1}\right.$ um $)$} \\
\cline { 2 - 3 } & $\begin{array}{c}\text { Single-layer } \\
\left(\eta_{S P \%}\right)\end{array}$ & $\begin{array}{c}\text { Multilayer } \\
\left(\eta_{S P} \%\right)\end{array}$ \\
\hline 1000 & 27.6 & 59.0 \\
1200 & 43.0 & 71.6 \\
1300 & 50.1 & 76 \\
1400 & 56.6 & 79.6 \\
1500 & 62.3 & 82.4 \\
\hline
\end{tabular}

\section{Conclusions}

In this article, we proposed and numerically analyzed a multilayer optical selective emitter for TPV applications, based on a metamaterial design. The metamaterial exhibited polarization and azimuthal angle independent selective emission. We considered InGaAs with energy band gap of $0.6 \mathrm{eV}$ and a cut-off wavelength of $2.1 \mu \mathrm{m}$. Geometric parameters such as the radius of the nano-rings, the number of layers, and the thicknesses of the thin films were studied and optimized to achieve perfect broadband emissivity above the band gap energy. The proposed metamaterial increased the efficiency of the TPV system. Efficiency was also enhanced when all radiated photons were absorbed by the TPV cell. The proposed multilayer exhibited an enhanced near perfect broadband and a wide-angle average emissivity of above $96.6 \%$ between the wavelengths of $0.2 \mu \mathrm{m}$ to $2.1 \mu \mathrm{m}$ on optimization of the geometric parameters. Furthermore, $79.6 \%$ spectral efficiency was obtained 
at the cut-off wavelength of InGaAs of $2.1 \mu \mathrm{m}$ and at an emitter temperature of $1400 \mathrm{~K}$. This result contributed to the enhancement of the TPV performance.

Author Contributions: F.T.M. is the author who made substantial contributions to investigation, design and writing-Original draft preparation. He was the core participant in every step. N.L., G.C., T.K., and H.H.C. are the authors who gave vital guidance, writing review, editing and gave very important advice when writing. H.H.C. is the supervisors of authors and he gave substantial suggestions during writing and all of other authors reviewed the manuscript.

Acknowledgments: This work was supported by the Center for Advanced MetaMaterials (CAMM) funded by the Minis-try of Science, ICT and Future Planning as Global Frontier Project (CAMM-No. NRF2014M3A6B3063716) and the Human Resources Development program (No. 20174030201720) of the Korea Institute of Energy Technology Evaluation and Planning (KETEP), grant funded by the Korea government Ministry of Trade, Industry and Energy.

Conflicts of Interest: The authors declare no conflict of interest.

\section{References}

1. Jin, S.; Lim, M.; Lee, S.S.; Lee, B.J. Hyperbolic metamaterial-based near-field thermophotovoltaic system for hundreds of nanometer vacuum gap. Opt. Express 2016, 24, A635-A649. [CrossRef] [PubMed]

2. Woolf, D.N.; Kadlec, E.A.; Bethke, D.; Grine, A.D.; Nogan, J.J.; Cederberg, J.G.; Hensley, J.M. High-efficiency thermophotovoltaic energy conversion enabled by a metamaterial selective emitter. Optica 2018, 5, $213-218$. [CrossRef]

3. Ghanekar, A.; Lin, L.; Zheng, Y. Novel and efficient Mie-metamaterial thermal emitter for thermophotovoltaic systems. Opt. Express 2016, 24, A868-A877. [CrossRef] [PubMed]

4. Tong, J.K.; Hsu, W.-C.H.; Huang, Y.; Boriskina, S.V.; Chen, G. Thin-film 'Thermal Well' Emitters and Absorbers for High-Efficiency Thermophotovoltaics. Sci. Rep. 2015, 5. [CrossRef] [PubMed]

5. Nguyen-Huu, N.; Pistora, J.; Cada, M. Wavelength-selective emitters with pyramid nanogratings enhanced by multiple resonance modes. Nanotechnology 2016, 27. [CrossRef] [PubMed]

6. Song, J.; $\mathrm{Wu}, \mathrm{H}$.; Cheng, Q.; Zhao, J. 1D trilayer films grating with $\mathrm{W} / \mathrm{SiO} 2 / \mathrm{W}$ structure as a wavelength-selective emitter for thermophotovoltaic applications. J. Quant. Spectrosc. Radiat. Transf. 2015, 158, 136-144. [CrossRef]

7. Wang, L.P.; Zhang, Z.M. Wavelength-selective and diffuse emitter enhanced by magnetic polaritons for thermophotovoltaics. Appl. Phys. Lett. 2012, 100. [CrossRef]

8. Song, J.; Si, M.; Cheng, Q.; Luo, Z. Two-dimensional trilayer grating with a metal/insulator/metal structure as a thermophotovoltaic emitter. Appl. Opt. 2016, 55, 1284-1290. [CrossRef] [PubMed]

9. Lin, S.Y.; Moreno, J.; Fleming, J.G. Three-dimensional photonic-crystal emitter for thermal photovoltaic power generation. Appl. Phys. Lett. 2003, 83, 380-382. [CrossRef]

10. Fleming, J.G. Addendum "Three-dimensional photonic-crystal emitter for thermal photovoltaic power generation" (vol 83, pg 380, 2003). Appl. Phys. Lett. 2005, 86, 380. [CrossRef]

11. Trupke, T.; Wurfel, P.; Green, M.A. Comment on "Three-dimensional photonic-crystal emitter for thermal photovoltaic power generation" [Appl. Phys. Lett., 83, 380 (2003)]. Appl. Phys. Lett. 2004, 84, 1997-1998. [CrossRef]

12. Hedayati, M.K.; Faupel, F.; Elbahri, M. Review of Plasmonic Nanocomposite Metamaterial Absorber. Materials 2014, 7, 1221-1248. [CrossRef] [PubMed]

13. Cui, Y.; He, Y.; Jin, Y.; Ding, F.; Yang, L.; Ye, Y.; Zhong, S.; Lin, Y.; He, S. Plasmonic and metamaterial structures as electromagnetic absorbers. Laser Photonics Rev. 2014, 8, 495-520. [CrossRef]

14. Yan, M.; Dai, J.; Qiu, M. Lithography-free broadband visible light absorber based on a mono-layer of gold nanoparticles. J. Opt. 2014, 16. [CrossRef]

15. Shemelya, C.; Meo, D.D.; Latham, N.P.; Wu, X.; Bingham, C.; Padilla, W.; Vandervelde, T.E. Stable high temperature metamaterial emitters for thermophotovoltaic applications. Appl. Phys. Lett. 2014, 104. [CrossRef]

16. Zhao, B.; Wanga, L.; Shuai, Y.; Zhang, Z.M. Thermophotovoltaic emitters based on a two-dimensional grating/thin-film nanostructure. Int. J. Heat Mass Transf. 2013, 67, 637-645. [CrossRef]

17. Cao, T.; Wang, S.; Wei, C.W. Simulation of tunable metamaterial perfect absorber by modulating $\mathrm{Bi}_{2} \mathrm{Se}_{3}$ dielectric function. Mater. Express 2016, 6, 45-52. [CrossRef]

18. Wang, H.; Chang, J.-Y.; Yang, Y.; Wang, L. Performance analysis of solar thermophotovoltaic conversion enhanced by selective metamaterial absorbers and emitters. Int. J. Heat Mass Transf. 2016, 98, 788-798. [CrossRef] 
19. Liu, X.L.; Tyler, T.; Starr, T.; Starr, A.F.; Jokerst, N.M.; Padilla, W.J. Taming the Blackbody with Infrared Metamaterials as Selective Thermal Emitters. Phys. Rev. Lett. 2011, 107. [CrossRef] [PubMed]

20. Woolf, D.; Hensley, J.; Cederberg, J.G.; Bethke, D.T.; Grine, A.D.; Shaner, E.A. Heterogeneous metasurface for high temperature selective emission. Appl. Phys. Lett. 2014, 105. [CrossRef]

21. Wang, H.; Chen, Q.; Wen, L.; Song, S.; Hu, X.; Xu, G. Titanium-nitride-based integrated plasmonic absorber/emitter for solar thermophotovoltaic application. Photonics Res. 2015, 3, 329-334. [CrossRef]

22. Gu, W.; Tang, G.; Tao, W. High efficiency thermophotovoltaic emitter by metamaterial-based nano-pyramid array. Opt. Express 2015, 23, 30681-30694. [CrossRef] [PubMed]

23. Rephaeli, E.; Fan, S.H. Absorber and emitter for solar thermophotovoltaic systems to achieve efficiency exceeding the Shockley-Queisser limit. Opt. Express 2009, 17, 15145-15159. [CrossRef] [PubMed]

24. Ding, F;; Cui, Y.; Ge, X.; Jin, Y.; He, S. Ultra-broadband microwave metamaterial absorber. Appl. Phys. Lett. $2012,100$. [CrossRef]

25. Wang, H.; Sivan, V.P.; Mitchell, A.; Rosengarten, G.; Phelan, P.; Wang, L. Highly efficient selective metamaterial absorber for high-temperature solar thermal energy harvesting. Sol. Energy Mater. Sol. Cells 2015, 137, $235-242$. [CrossRef]

26. Molesky, S.; Dewalt, C.J.; Jacob, Z. High temperature epsilon-near-zero and epsilon-near-pole metamaterial emitters for thermophotovoltaics. Opt. Express 2013, 21, A96-A110. [CrossRef] [PubMed]

27. Deng, H.; Wang, T.; Gao, J.; Yang, X. Metamaterial thermal emitters based on nanowire cavities for high-efficiency thermophotovoltaics. J. Opt. 2014, 16. [CrossRef]

28. Yun, D.; Min, X.Y. Perfect solar absorber based on nanocone structured surface for high-efficiency solar thermoelectric generators. Sci. China-Technol. Sci. 2015, 58, 19-28.

29. Wen, D.; Yang, H.; Ye, Q.; Li, M.; Guo, L.; Zhang, J. Broadband metamaterial absorber based on a multi-layer structure. Phys. Scr. 2013, 88. [CrossRef]

30. Adachi, S. Aluminum Nitride (AIN). In Optical Constants of Crystalline and Amorphous Semiconductors; Springer: New York, NY, USA, 1999; pp. 143-151.

31. Wu, C.; Neuner, B., III; John, J.; Milder, A.; Zollars, B.; Savoy, S.; Shvets, G. Metamaterial-based integrated plasmonic absorber/emitter for solar thermo-photovoltaic systems. J. Opt. 2012, 14, 024005. [CrossRef]

32. Rakic', A.D.; Djurisic, A.B.; Elazar, J.M.; Majewski, M.L. Optical properties of metallic films for vertical-cavity optoelectronic devices. Appl. Opt. 1998, 37, 5271-5283. [CrossRef]

33. Pastrnak, J.; Roskovcova, L. Refraction index measurements on AlN single crystals. Phys. Status Solidi B-Basic Solid State 1966, 14. [CrossRef]

34. Hwanseong, L.; Taehwan, K.; Fekadu, T.M.; Cho, H.H. Enhancing radiative cooling performance using metal-dielectric-metal metamaterials. J. Mech. Sci. Technol. 2017, 31, 5107-5112.

35. Mitrofanov, O.; Dominec, F.; Kužel, P.; Reno, J.L.; Brener, I.; Chung, U.-C.; Elissalde, C.; Maglione, M.; Mounaix, P. Near-field probing of Mie resonances in single $\mathrm{TiO}_{2}$ microspheres at terahertz frequencies. Opt. Express 2014, 22, 23034-23042. [CrossRef] [PubMed]

36. Wang, H.; Wang, L. Perfect selective metamaterial solar absorbers. Opt. express 2013, 21, A1078-A1093. [CrossRef] [PubMed]

37. Ferrari, C.; Melino, F.; Pinelli, M.; Spina, P.R. Thermophotovoltaic energy conversion: Analytical aspects, prototypes and experiences. Appl. Energy 2014, 113, 1717-1730. [CrossRef]

38. Rinnerbauer, V.; Lenert, A.; Bierman, D.M.; Yeng, Y.X.; Chan, W.R.; Geil, R.D.; Senkevich, J.J. Metallic photonic crystal absorber-emitter for efficient spectral control in high-temperature solar thermophotovoltaics. Adv. Energy Mater. 2014, 4. [CrossRef]

(C) 2018 by the authors. Licensee MDPI, Basel, Switzerland. This article is an open access article distributed under the terms and conditions of the Creative Commons Attribution (CC BY) license (http://creativecommons.org/licenses/by/4.0/). 